\title{
Correction to: A Novel Fast and Secure Approach for Voice Encryption Based on DNA Computing
}

\author{
Hamidreza Kakaei Kate $\cdot$ Jafar Razmara $\cdot$ Ayaz Isazadeh
}

Published online: 16 June 2018

(C) 3D Research Center, Kwangwoon University and Springer-Verlag GmbH Germany, part of Springer Nature 2018

Correction to: 3D Res (2018) 9:17

https://doi.org/10.1007/s13319-018-0167-x

The original version of this article unfortunately contained mistakes in Table 5. In the column Correlation Encryption, Key space, SNR and PSNR was incorrect.

The corrected Table 5 is given below.

The original article has been corrected.

The original article can be found online at https:// doi.org/10.1007/s13319-018-0167-x.

H. Kakaei Kate · J. Razmara ( () ) A. Isazadeh Department of Computer Science, Faculty of Mathematical Sciences, University of Tabriz, Tabriz, Iran e-mail: razmara@tabrizu.ac.ir 
Table 5 Comparing the average of most common security parameters of the proposed approach and some other schemes

\begin{tabular}{|c|c|c|c|c|c|c|}
\hline Method & Authors & $\begin{array}{l}\text { Correlation } \\
\text { encryption }\end{array}$ & $\begin{array}{l}\text { Correlation } \\
\text { decryption }\end{array}$ & Key space & SNR & PSNR \\
\hline Transform domain & Mosa et al. [9] & NA & NA & $10^{126} \approx 8.5 * 10^{37}$ & NA & NA \\
\hline $\begin{array}{l}\text { Random Lorenz and DCT } \\
\text { permutation }\end{array}$ & Sakhan et al. [15] & NA & NA & $2^{144} \approx 2.3 * 10^{43}$ & NA & NA \\
\hline Confusion and diffusion & Liu et al. [16] & 0.0044 & 0.8335 & $2^{128} \approx 3.4 * 10^{38}$ & - & 4.426 \\
\hline Cosine number transform & Lima et al. [17] & 0.002 & 0.9445 & $2^{256} \approx 1.1 * 10^{77}$ & - & - \\
\hline Off-line ICA & Sadkhan et al. [18] & NA & NA & NA & 17.14 & - \\
\hline Shuffle encryption & Tamimi et al. [19] & 0.0263 & NA & NA & NA & 9.534 \\
\hline Compound chaotic mapping & Li et al. [20] & 0.0282 & 0.9927 & $2 * 10^{28}$ & NA & NA \\
\hline $\begin{array}{l}\text { Double dimension discrete- } \\
\text { time }\end{array}$ & Akgül et al. [22] & NA & NA & $10^{42}$ & NA & NA \\
\hline $\begin{array}{l}\text { Compressive sensing and } \\
\text { arnold transform }\end{array}$ & $\begin{array}{l}\text { Augustine et al. } \\
\text { [23] }\end{array}$ & 0.0014 & 0.8239 & $2^{128} \approx 3.4 * 10^{38}$ & -1.47 & NA \\
\hline Mixture chaos & $\begin{array}{l}\text { Gasemzadeh et al. } \\
\text { [24] }\end{array}$ & 0.0092 & 0.9505 & $2^{144} \approx 2.3 * 10^{43}$ & NA & NA \\
\hline PRBG & Sadkhan et al. [25] & NA & NA & $10^{112}$ & NA & NA \\
\hline Chaotic shift key & $\begin{array}{l}\text { Sathiyamurthi } \\
\text { et al. [26] }\end{array}$ & 0.0223 & 0.9995 & NA & 33.71 & 60.244 \\
\hline Software package & Eldin et al. [40] & 0.0280 & 0.9850 & NA & NA & NA \\
\hline AES & AES & 0.0097 & NA & $2^{256} \approx 1.1 * 10^{77}$ & -1.4 & - \\
\hline $\begin{array}{l}\text { DNA computing and } \\
\text { permutation coding }\end{array}$ & $\begin{array}{l}\text { Proposed } \\
\text { approach }\end{array}$ & -0.0009 & 1 & $2^{512} \approx 1.34 * 10^{154}$ & -30.93 & -7.80 \\
\hline
\end{tabular}

NA not available 